\title{
Current status of molecular biology and treatment strategy for neuroblastoma
}

\author{
Hideo Mugishima
}

Received: 24 April 2012/Published online: 16 May 2012

(C) Japan Society of Clinical Oncology 2012

Neuroblastomas are tumors of the sympathetic nervous system in childhood. For more than 30 years, neuroblastoma has remained one of the most challenging malignant tumors for both clinicians and basic scientists. Many advances have been made in understanding the oncogenesis and biology of neuroblastoma, and some of these advances may be translated into better clinical management. However, almost no improvement of survival rates has been achieved, at least for the large group of patients who have metastatic disease.

This is one of the reasons why neuroblastomas have been studied so extensively by pediatric oncologists worldwide. The majority of patients with neuroblastoma is categorized to high-risk groups based on age at diagnosis, stage, histology, MYCN status and DNA ploidy and their prognosis remains unsatisfactory; 5-year event-free survival (EFS) rate is generally $40 \%$.

To improve the prognosis of children with neuroblastoma, current research has focused on the following areas: (1) search for earlier diagnosis of the disease, (2) studies to detect minimal residual disease with the use of techniques such as immune cytology, scintigraphic approaches, and tumor markers, (3) attempts to change the biology of the disease, such as by artificial induction of regression and maturation processes and stimulation of immune response, by the use of retinoid compounds (13-cis-retinoic acid), monoclonal antibodies (anti-GD2), granulocyte-macrophage colony-stimulating factor, and interleukin 2, (4) definition of biologically more meaningful risk factors, (5) development of new therapeutic strategies, such as new combinations of high-dose chemotherapy and both autologous and allogeneic stem cell transplantation, and (6) design of targeted agents for destroying tumor cells specifically, such as $\mathrm{I}^{131}$-MIBG (metaiodobenzylguanidine) and monoclonal antibody-drug conjugates.

In terms of treatment, a large number of novel targeted agents such as anaplastic lymphoma kinase (ALK) inhibitor and aurora kinase A inhibitor are under development. Nowadays, comprehensive genome-wide characterization is being increasingly used to extensively profile individual tumors. Future treatment would seem to be individually planned, adapting targeted agents based on personal biological tumor characteristics.

There are still many questions to be answered in relation to the molecular pathogenesis and clinical treatment of neuroblastomas. Thus, it seemed timely to summarize the current state of the art of neuroblastoma biology and therapy. Dr. T. Kamijo and Dr. A. Nakagawara describe the topic of molecular and genetic bases of neuroblastoma and Dr. J. Hara introduces the development of treatment strategy for neuroblastoma.

We hope this review article will be helpful for understanding the mechanism of neuroblastoma tumorigenesis and aggressiveness and for developing a new therapeutic stratification for neuroblastoma.

Conflict of interest The author declares that he has no conflict of interest.

H. Mugishima $(\bowtie)$

Department of Pediatrics and Child Health, Nihon University

School of Medicine, Tokyo, Japan

e-mail: mugishima.hideo@nihon-u.ac.jp 